Volume 70, Number 2, Pages 622-630(2021)

DOI: $10.31801 /$ cfsuasmas. 854761

ISSN 1303-5991 E-ISSN 2618-6470

Received by the editors: January 5, 2021; Accepted: February 17, 2021

\title{
THE GENERALIZED LUCAS HYBRINOMIALS WITH TWO VARIABLES
}

\author{
Emre SEVGİ \\ Department of Mathematics, Gazi University, Ankara, TURKEY
}

\begin{abstract}
. Özdemir defined the hybrid numbers as a generalization of complex, hyperbolic and dual numbers. In this research, we define the generalized Lucas hybrinomials with two variables. Also, we get the Binet formula, generating function and some properties for the generalized Lucas hybrinomials. Moreover, Catalan's, Cassini's and d'Ocagne's identities for these hybrinomials are obtained. Lastly, by the help of the matrix theory we derive the matrix representation of the generalized Lucas hybrinomials.
\end{abstract}

\section{Introduction}

Many researchers have studied on applications of the Fibonacci and the Lucas numbers for a long time in engineering, arts, physics and nature. These sequences have taken a huge interest of many authors.

The Fibonacci numbers are defined recursively by

$$
F_{n}=F_{n-1}+F_{n-2}
$$

for $n \geq 2$ with initial values $F_{0}=0$ and $F_{1}=14$.

The Lucas numbers are defined with the same recurrence relation of the Fibonacci numbers with initial values $L_{0}=2$ and $L_{1}=1$ [4].

For the variable $x$, Catalan defined the Lucas polynomials with the recurrence relation

$$
L_{n}(x)=x L_{n-1}(x)+L_{n-2}(x), n \geq 2
$$

with $L_{0}(x)=2$ and $L_{1}(x)=x[4$.

Bergum and Hoggatt studied on the generalized Lucas polynomials and defined these polynomials recursively by

2020 Mathematics Subject Classification. Primary 11B37, 11B39.

Keywords and phrases. Hybrid numbers, Fibonacci numbers, Lucas numbers, polynomials, hybrinomials, recurrence relation.

$$
\text { 凶emresevgi@gazi.edu.tr }
$$

(D) 0000-0003-2711-9880.

(C)2021 Ankara University Communications Faculty of Sciences University of Ankara-Series A1 Mathematics and Statistics 


$$
V_{n}(x, y)=x V_{n-1}(x, y)+y V_{n-2}(x, y), n \geq 2
$$

with the initial conditions $V_{0}(x, y)=2$ and $V_{1}(x, y)=x$ [1].

After that, Swamy obtained some identities and properties for the generalized Lucas sequence 7 .

The first few terms of this sequence are

$$
\begin{array}{lc}
n & V_{n}(x, y) \\
0 & 2 \\
1 & x \\
2 & x^{2}+2 y \\
3 & x^{3}+3 x y \\
4 & x^{4}+4 x^{2} y+2 y^{2} \\
5 & x^{5}+5 x^{3} y+5 x y^{2} \\
6 & x^{6}+6 x^{4} y+9 x^{2} y^{2}+2 y^{3}
\end{array}
$$

For simplicity, we will use $V_{n}$ instead of $V_{n}(x, y)$.

The characteristic equation of this sequence is

$$
v^{2}-x v-y=0
$$

with the roots

$$
\alpha=\frac{x+\sqrt{x^{2}+4 y}}{2}, \beta=\frac{x-\sqrt{x^{2}+4 y}}{2} .
$$

Lemma 1. [7] The roots $\alpha$ and $\beta$ defined in (1] satisfy the following properties

- $\alpha+\beta=x$

- $\alpha-\beta=\sqrt{x^{2}+4 y}$

- $\alpha \beta=-y$

Lemma 2. [7] For $n \geq 0$ the Binet formula for the generalized Lucas polynomials is

$$
V_{n}=\alpha^{n}+\beta^{n} \text {. }
$$

The hybrid numbers were defined by Özdemir as a generalization of complex, hyperbolic and dual numbers [5]. The set of hybrid numbers is

$$
K=\{a+b i+c \varepsilon+d h: a, b, c, d \in \mathbb{R}\} .
$$

Let $Z_{1}=a_{1}+b_{1} i+c_{1} \varepsilon+d_{1} h$ and $Z_{2}=a_{2}+b_{2} i+c_{2} \varepsilon+d_{2} h$ be any two hybrid numbers. Then the main operations on hybrid numbers are defined as follows:

$$
\begin{aligned}
& Z_{1}=Z_{2} \text { if and only if } a_{1}=a_{2}, b_{1}=b_{2}, c_{1}=c_{2}, d_{1}=d_{2} \\
& Z_{1}+Z_{2}=\left(a_{1}+a_{2}\right)+\left(b_{1}+b_{2}\right) i+\left(c_{1}+c_{2}\right) \varepsilon+\left(d_{1}+d_{2}\right) h \\
& Z_{1}-Z_{2}=\left(a_{1}-a_{2}\right)+\left(b_{1}-b_{2}\right) i+\left(c_{1}-c_{2}\right) \varepsilon+\left(d_{1}-d_{2}\right) h
\end{aligned}
$$


$s Z_{1}=s a_{1}+s b_{1} i+s c_{1} \varepsilon+s d_{1} h$, where $s \in \mathbb{R}$.

By using the following multiplication table, one can find the product of any two hybrid numbers:

\begin{tabular}{c|c|c|c|c}
$\cdot$ & 1 & $i$ & $\varepsilon$ & $h$ \\
\hline 1 & 1 & $i$ & $\varepsilon$ & $h$ \\
\hline$i$ & $i$ & -1 & $1-h$ & $\varepsilon+i$ \\
\hline$\varepsilon$ & $\varepsilon$ & $h+1$ & 0 & $-\varepsilon$ \\
\hline$h$ & $h$ & $-\varepsilon-i$ & $\varepsilon$ & 1
\end{tabular}

By using the elements of integer sequences as coefficients of hybrid numbers, many authors defined new type of hybrid numbers ( 8, 9, 3, 2, 10, 11, 12,14). The most exciting studies among the cited works are the Fibonacci hybrid numbers and the Lucas hybrid numbers which were defined as

$$
F H_{n}=F_{n}+i F_{n+1}+\varepsilon F_{n+2}+h F_{n+3}
$$

and

respectively.

$$
L H_{n}=L_{n}+i L_{n+1}+\varepsilon L_{n+2}+h L_{n+3}
$$

After that, for $n \geq 0$ Szynal-Liana and Włoch $[13$ defined the Fibonacci and the Lucas hybrinomials as

$$
F H_{n}(x)=F_{n}(x)+i F_{n+1}(x)+\varepsilon F_{n+2}(x)+h F_{n+3}(x)
$$

and

$$
L H_{n}(x)=L_{n}(x)+i L_{n+1}(x)+\varepsilon L_{n+2}(x)+h L_{n+3}(x)
$$

respectively.

\section{Main Results}

Definition 3. For $n \geq 0$ the Lucas hybrinomials with two variables $x$ and $y$, called the generalized Lucas hybrinomials defined by

$$
V H_{n}(x, y)=V_{n}+i V_{n+1}+\varepsilon V_{n+2}+h V_{n+3}
$$

where $V_{n}$ is the nth generalized Lucas polynomial.

For simplicity, we will use $V H_{n}$ instead of $V H_{n}(x, y)$.

Theorem 4. For the variables $x$ and $y$, the generalized Lucas hybrinomials provides the recurrence relation

$$
V H_{n}=x V H_{n-1}+y V H_{n-2}, n \geq 2
$$

with the initial conditions

$$
\begin{aligned}
& V H_{0}=2+i x+\varepsilon\left(x^{2}+2 y\right)+h\left(x^{3}+3 x y\right) \text { and } \\
& V H_{1}=x+i\left(x^{2}+2 y\right)+\varepsilon\left(x^{3}+3 x y\right)+h\left(x^{4}+4 x^{2} y+2 y^{2}\right) .
\end{aligned}
$$


Proof. For $n=2$, we get

$$
\begin{aligned}
V H_{2}= & x V H_{1}+y V H_{0} \\
= & x\left(x+i\left(x^{2}+2 y\right)+\varepsilon\left(x^{3}+3 x y\right)+h\left(x^{4}+4 x^{2} y+2 y^{2}\right)\right) \\
& +y\left(2+i x+\varepsilon\left(x^{2}+2 y\right)+h\left(x^{3}+3 x y\right)\right) \\
= & x^{2}+2 y+i\left(x^{3}+3 x y\right)+\varepsilon\left(x^{4}+4 x^{2} y+2 y^{2}\right)+h\left(x^{5}+5 x^{3} y+5 x y^{2}\right) \\
= & V_{2}+i V_{3}+\varepsilon V_{4}+h V_{5} .
\end{aligned}
$$

For $n>2$, using the definition of the generalized Lucas polynomials, we obtain

$$
\begin{aligned}
V H_{n}= & V_{n}+i V_{n+1}+\varepsilon V_{n+2}+h V_{n+3} \\
= & \left(x V_{n-1}+y V_{n-2}\right)+i\left(x V_{n}+y V_{n-1}\right) \\
& +\varepsilon\left(x V_{n+1}+y V_{n}\right)+h\left(x V_{n+2}+y V_{n+1}\right) \\
= & x\left(V_{n-1}+i V_{n}+\varepsilon V_{n+1}+h V_{n+2}\right) \\
& +y\left(V_{n-2}+i V_{n-1}+\varepsilon V_{n}+h V_{n+1}\right) \\
= & x V H_{n-1}+y V H_{n-2} .
\end{aligned}
$$

So, the proof is completed.

For $y=1$, we obtain the Lucas hybrinomials.

For $x=y=1$, we obtain the Lucas hybrid numbers.

Theorem 5. For any integer $n \geq 0$, the Binet formula for the generalized Lucas hybrinomials is defined as

$$
\begin{aligned}
& V H_{n}=\alpha^{n}\left(1+i \alpha+\varepsilon \alpha^{2}+h \alpha^{3}\right)+\beta^{n}\left(1+i \beta+\varepsilon \beta^{2}+h \beta^{3}\right) \\
& \text { where } \alpha=\frac{x+\sqrt{x^{2}+4 y}}{2} \text { and } \beta=\frac{x-\sqrt{x^{2}+4 y}}{2} .
\end{aligned}
$$

Proof. Using the definition of the generalized Lucas hybrinomials and the Binet formula for the generalized Lucas polynomials, we get

$$
\begin{aligned}
V H_{n} & =V_{n}+i V_{n+1}+\varepsilon V_{n+2}+h V_{n+3} \\
& =\alpha^{n}+\beta^{n}+i\left(\alpha^{n+1}+\beta^{n+1}\right)+\varepsilon\left(\alpha^{n+2}+\beta^{n+2}\right)+h\left(\alpha^{n+3}+\beta^{n+3}\right) \\
& =\alpha^{n}\left(1+i \alpha+\varepsilon \alpha^{2}+h \alpha^{3}\right)+\beta^{n}\left(1+i \beta+\varepsilon \beta^{2}+h \beta^{3}\right)
\end{aligned}
$$

For expressing the notations simply, let

$$
\begin{aligned}
& \widehat{\alpha}=1+i \alpha+\varepsilon \alpha^{2}+h \alpha^{3} \\
& \widehat{\beta}=1+i \beta+\varepsilon \beta^{2}+h \beta^{3} .
\end{aligned}
$$

Then, we can write the Binet formula for the generalized Lucas hybrinomials as

$$
V H_{n}=\alpha^{n} \widehat{\alpha}+\beta^{n} \widehat{\beta} .
$$

Theorem 6. The generating function for the generalized Lucas hybrinomials is 


$$
\begin{aligned}
& \sum_{n=0}^{\infty} V H_{n} t^{n} \\
& \quad=\frac{2+i x+\varepsilon\left(x^{2}+2 y\right)+h\left(x^{3}+3 x y\right)+\left(-x+i 2 y+\varepsilon x y+h\left(x^{2} y+2 y^{2}\right)\right) t}{1-x t-y t^{2}} .
\end{aligned}
$$

Proof. Suppose that the formal power series representation of the generating function for the generalized Lucas hybrinomials is

$$
G(t)=\sum_{n=0}^{\infty} V H_{n} t^{n}=V H_{0}+V H_{1} t+V H_{2} t^{2}+\cdots
$$

Then, multiplying the equation $(2)$ by $-x t$ and $-y t^{2}$ respectively, we have $-G(t) x t=-V H_{0} x t-V H_{1} x t^{2}-V H_{2} x t^{3}-\cdots$

and

$$
-G(t) y t^{2}=-V H_{0} y t^{2}-V H_{1} y t^{3}-V H_{2} y t^{4}-\cdots
$$

By using the above equations and the fact that for $n \geq 2$ the coefficients of $t^{n}$ are zero by the recurrence relation of the generalized Lucas hybrinomials, we obtain

$$
G(t)\left(1-x t-y t^{2}\right)=V H_{0}+\left(V H_{1}-V H_{0} x\right) t .
$$

Finally, by substituting $V H_{0}$ and $V H_{1}$ in the equation (3), we get

$$
G(t)=\frac{2+i x+\varepsilon\left(x^{2}+2 y\right)+h\left(x^{3}+3 x y\right)+\left(-x+i 2 y+\varepsilon x y+h\left(x^{2} y+2 y^{2}\right)\right) t}{1-x t-y t^{2}} .
$$

Lemma 7. [6] For any integer $n \geq 2$, the generalized Lucas polynomials provides the summation formula

$$
\sum_{m=1}^{n-1} V_{m}=\frac{V_{n}+y V_{n-1}-x-2 y}{x+y-1} .
$$

Theorem 8. For any integer $n \geq 2$, the generalized Lucas hybrinomials provides the summation formula

$$
\sum_{m=1}^{n-1} V H_{m}=\frac{V H_{n}+y V H_{n-1}-V H_{1}-y V H_{0}}{x+y-1} .
$$

Proof. By using the definition of the generalized Lucas hybrinomials, we have

$$
\begin{aligned}
\sum_{m=1}^{n-1} V H_{m}= & V H_{1}+V H_{2}+\cdots+V H_{n-1} \\
= & V_{1}+i V_{2}+\varepsilon V_{3}+h V_{4} \\
& +V_{2}+i V_{3}+\varepsilon V_{4}+h V_{5}
\end{aligned}
$$




$$
\begin{aligned}
& +V_{n-1}+i V_{n}+\varepsilon V_{n+1}+h V_{n+2} \\
= & V_{1}+V_{2}+\cdots+V_{n-1} \\
& +i\left[V_{2}+V_{3}+\cdots+V_{n}+\left(V_{1}-V_{1}\right)\right] \\
& +\varepsilon\left[V_{3}+V_{4}+\cdots+V_{n+1}+\left(V_{1}+V_{2}-V_{1}-V_{2}\right)\right] \\
& +h\left[V_{4}+V_{5}+\cdots+V_{n+2}+\left(V_{1}+V_{2}+V_{3}-V_{1}-V_{2}-V_{3}\right)\right]
\end{aligned}
$$

By using the previous lemma, we have

$$
\begin{aligned}
\sum_{m=1}^{n-1} V H_{m}= & \frac{V_{n}+y V_{n-1}-x-2 y}{x+y-1} \\
& +i\left(\frac{V_{n+1}+y V_{n}-x-2 y}{x+y-1}-V_{1}\right) \\
& +\varepsilon\left(\frac{V_{n+2}+y V_{n+1}-x-2 y}{x+y-1}-V_{1}-V_{2}\right) \\
& +h\left(\frac{V_{n+3}+y V_{n+2}-x-2 y}{x+y-1}-V_{1}-V_{2}-V_{3}\right) .
\end{aligned}
$$

Substituting $V_{1}, V_{2}, V_{3}$ and making the fractions common denominator, we obtain

$$
\begin{aligned}
\sum_{m=1}^{n-1} V H_{m}= & \frac{V_{n}+y V_{n-1}-x-2 y}{x+y-1} \\
& +i\left(\frac{V_{n+1}+y V_{n}-x-2 y-x(x+y-1)}{x+y-1}\right) \\
+ & \varepsilon \frac{1}{x+y-1}\left(V_{n+2}+y V_{n+1}-x-2 y-x(x+y-1)\right. \\
& \left.\quad-\left(x^{2}+2 y\right)(x+y-1)\right) \\
+ & h \frac{1}{x+y-1}\left(V_{n+3}+y V_{n+2}-x-2 y-x(x+y-1)\right. \\
& \left.\quad-\left(x^{2}+2 y\right)(x+y-1)-\left(x^{3}+3 x y\right)(x+y-1)\right) .
\end{aligned}
$$

Finally, we get the result as

$$
\begin{aligned}
\sum_{m=1}^{n-1} V H_{m}= & \frac{V_{n}+i V_{n+1}+\varepsilon V_{n+2}+h V_{n+3}}{x+y-1} \\
& +y \frac{V_{n-1}+i V_{n}+\varepsilon V_{n+1}+h V_{n+2}}{x+y-1} \\
& -\frac{1}{x+y-1}\left(x+2 y+i\left(x^{2}+2 y+y x\right)+\varepsilon\left(x^{3}+3 x y+y\left(x^{2}+2 y\right)\right)\right. \\
& \left.+h\left(x^{4}+4 x^{2} y+2 y^{2}+y\left(x^{3}+3 x y\right)\right)\right)
\end{aligned}
$$




$$
=\frac{V H_{n}+y V H_{n-1}-V H_{1}-y V H_{0}}{x+y-1} .
$$

Theorem 9 (Catalan Identity). For the nonnegative integers $n$ and $r$ with $n \geq r$, we have

$$
V H_{n-r} V H_{n+r}-\left(V H_{n}\right)^{2}=(-y)^{n} \widehat{\alpha} \widehat{\beta}\left(\frac{\beta^{r}}{\alpha^{r}}-1\right)+(-y)^{n} \widehat{\beta} \widehat{\alpha}\left(\frac{\alpha^{r}}{\beta^{r}}-1\right) .
$$

Proof. By using the Binet formula for the generalized Lucas hybrinomials, we have

$$
\begin{array}{rl}
V H_{n-r} & V H_{n+r}-\left(V H_{n}\right)^{2} \\
& =\left(\alpha^{n-r} \widehat{\alpha}+\beta^{n-r} \widehat{\beta}\right)\left(\alpha^{n+r} \widehat{\alpha}+\beta^{n+r} \widehat{\beta}\right)-\left(\alpha^{n} \widehat{\alpha}+\beta^{n} \widehat{\beta}\right)\left(\alpha^{n} \widehat{\alpha}+\beta^{n} \widehat{\beta}\right) \\
& =\alpha^{n-r} \beta^{n+r} \widehat{\alpha} \widehat{\beta}+\beta^{n-r} \alpha^{n+r} \widehat{\beta} \widehat{\alpha}-\alpha^{n} \beta^{n} \widehat{\alpha} \widehat{\beta}-\beta^{n} \alpha^{n} \widehat{\beta} \widehat{\alpha} \\
& =\alpha^{n} \beta^{n} \widehat{\alpha} \widehat{\beta}\left(\frac{\beta^{r}}{\alpha^{r}}-1\right)+\beta^{n} \alpha^{n} \widehat{\beta} \widehat{\alpha}\left(\frac{\alpha^{r}}{\beta^{r}}-1\right) \\
& =(-y)^{n} \widehat{\alpha} \widehat{\beta}\left(\frac{\beta^{r}}{\alpha^{r}}-1\right)+(-y)^{n} \widehat{\beta} \widehat{\alpha}\left(\frac{\alpha^{r}}{\beta^{r}}-1\right) .
\end{array}
$$

Theorem 10 (Cassini Identity). For any nonnegative integer $n$, we have

$$
V H_{n-1} V H_{n+1}-\left(V H_{n}\right)^{2}=(-y)^{n} \widehat{\alpha} \widehat{\beta}\left(\frac{\beta}{\alpha}-1\right)+(-y)^{n} \widehat{\beta} \widehat{\alpha}\left(\frac{\alpha}{\beta}-1\right)
$$

Proof. Since the Cassini identity is a special case of the Catalan identity, by taking $r=1$ in the Catalan identity theorem can be proved easily.

Theorem 11 (d'Ocagne Identity). For the nonnegative integers $m$ and $n$ with $m \geq n$, we have

$$
V H_{m} V H_{n+1}-V H_{m+1} V H_{n}=(-y)^{n}(\alpha-\beta)\left(\beta^{m-n} \widehat{\beta} \widehat{\alpha}-\alpha^{m-n} \widehat{\alpha} \widehat{\beta}\right) .
$$

Proof. By using the Binet formula for the generalized Lucas hybrinomials, we have

$$
\begin{aligned}
& V H_{m} V H_{n+1}-V H_{m+1} V H_{n} \\
&=\left(\alpha^{m} \widehat{\alpha}+\beta^{m} \widehat{\beta}\right)\left(\alpha^{n+1} \widehat{\alpha}+\beta^{n+1} \widehat{\beta}\right)-\left(\alpha^{m+1} \widehat{\alpha}+\beta^{m+1} \widehat{\beta}\right)\left(\alpha^{n} \widehat{\alpha}+\beta^{n} \widehat{\beta}\right) \\
&=\alpha^{m+n+1} \widehat{\alpha}^{2}+\alpha^{m} \beta^{n+1} \widehat{\alpha} \widehat{\beta}+\beta^{m} \alpha^{n+1} \widehat{\beta} \widehat{\alpha}+\beta^{m+n+1} \widehat{\beta}^{2} \\
&-\alpha^{m+n+1} \widehat{\alpha}^{2}-\alpha^{m+1} \beta^{n} \widehat{\alpha} \widehat{\beta}-\beta^{m+1} \alpha^{n} \widehat{\beta} \widehat{\alpha}-\beta^{m+n+1} \widehat{\beta}^{2} \\
&=\left(\alpha^{m} \beta^{n+1}-\alpha^{m+1} \beta^{n}\right) \widehat{\alpha} \widehat{\beta}+\left(\beta^{m} \alpha^{n+1}-\beta^{m+1} \alpha^{n}\right) \widehat{\beta} \widehat{\alpha} \\
&= \alpha^{m} \beta^{n}(\beta-\alpha) \widehat{\alpha} \widehat{\beta}+\beta^{m} \alpha^{n}(\alpha-\beta) \widehat{\beta} \widehat{\alpha} \\
&=(-y)^{n}(\alpha-\beta)\left(\beta^{m-n} \widehat{\beta} \widehat{\alpha}-\alpha^{m-n} \widehat{\alpha} \widehat{\beta}\right) .
\end{aligned}
$$


Theorem 12. For any nonnegative integer n, we have

$$
\left[\begin{array}{cc}
V H_{n+2} & V H_{n+1} \\
V H_{n+1} & V H_{n}
\end{array}\right]=\left[\begin{array}{cc}
V H_{2} & V H_{1} \\
V H_{1} & V H_{0}
\end{array}\right]\left[\begin{array}{ll}
x & 1 \\
y & 0
\end{array}\right]^{n}
$$

Proof. We prove the theorem using induction method on $n$.

For $n=0$, the result is obvious.

Assume that for any $n \geq 0$ the theorem holds

$$
\left[\begin{array}{cc}
V H_{n+2} & V H_{n+1} \\
V H_{n+1} & V H_{n}
\end{array}\right]=\left[\begin{array}{cc}
V H_{2} & V H_{1} \\
V H_{1} & V H_{0}
\end{array}\right]\left[\begin{array}{ll}
x & 1 \\
y & 0
\end{array}\right]^{n} .
$$

We must show that for $n+1$ the theorem holds

$$
\left[\begin{array}{ll}
V H_{n+3} & V H_{n+2} \\
V H_{n+2} & V H_{n+1}
\end{array}\right]=\left[\begin{array}{ll}
V H_{2} & V H_{1} \\
V H_{1} & V H_{0}
\end{array}\right]\left[\begin{array}{ll}
x & 1 \\
y & 0
\end{array}\right]^{n+1} .
$$

By using the induction hypothesis, we have

$$
\begin{aligned}
{\left[\begin{array}{ll}
V H_{2} & V H_{1} \\
V H_{1} & V H_{0}
\end{array}\right]\left[\begin{array}{ll}
x & 1 \\
y & 0
\end{array}\right]^{n+1} } & =\left[\begin{array}{cc}
V H_{2} & V H_{1} \\
V H_{1} & V H_{0}
\end{array}\right]\left[\begin{array}{ll}
x & 1 \\
y & 0
\end{array}\right]^{n}\left[\begin{array}{ll}
x & 1 \\
y & 0
\end{array}\right] \\
& =\left[\begin{array}{ll}
V H_{n+2} & V H_{n+1} \\
V H_{n+1} & V H_{n}
\end{array}\right]\left[\begin{array}{cc}
x & 1 \\
y & 0
\end{array}\right] \\
& =\left[\begin{array}{cc}
x V H_{n+2}+y V H_{n+1} & V H_{n+2} \\
x V H_{n+1}+y V H_{n} & V H_{n+1}
\end{array}\right] \\
& =\left[\begin{array}{ll}
V H_{n+3} & V H_{n+2} \\
V H_{n+2} & V H_{n+1}
\end{array}\right]
\end{aligned}
$$

which completes the proof.

\section{Conclusion}

In the present work, we define the generalized Lucas hybrinomials with two variables $x$ and $y$. Then, Binet formula, generating function and some properties of the generalized Lucas hybrinomials are obtained. Moreover, we obtain Catalan, Cassini and d'Ocagne identities for these hybrinomials. Finally, we derive the generalized Lucas hybrinomials by the help of matrix theory.

Declaration of Competing Interests The author declare that they have no known competing financial interest or personal relationship that could have appeared to influence the work reported in this paper.

Acknowledgments The author would like to thank the reviewer for his/her valuable comments and suggestions to improve the quality of the paper. 


\section{REFERENCES}

[1] Bergum, G.E., Hoggatt Jr., V.E., Irreducibility of Lucas and generalized Lucas polynomials, Fibonacci Quarterly, 12(1) (1974), 95-100.

[2] Catarino, P., On $k$-Pell hybrid numbers, Journal of Discrete Mathematical Sciences and Cryptography, 22(1) (2019), 83-89. https://doi.org/10.1080/09720529.2019.1569822

[3] Kizılateş, C., A New Generalization of Fibonacci hybrid and Lucas hybrid numbers, Chaos, Solitons and Fractals, 130 (2020).https://doi.org/10.1016/j.chaos.2019.109449

[4] Koshy, T., Fibonacci and Lucas Numbers with Applications, John Wiley and Sons Inc., New York, 2001.

[5] Özdemir, M., Introduction to hybrid numbers, Advances in Applied Clifford Algebras, 28 (2018). https://doi.org/10.1007/s00006-018-0833-3

[6] Panwar, Y. K., Singh, M., Generalized bivariate Fibonacci-like polynomials, International Journal of Pure Mathematics, 1 (2014), 8-13.

[7] Swamy, M.N.S., Generalized Fibonacci and Lucas polynomials, and their associated diagonal polynomials, Fibonacci Quarterly, 37 (1999), 213-222.

[8] Szynal-Liana, A., Włoch, I., On Jacobsthal and Jacobsthal-Lucas hybrid numbers, Annales Mathematicae Silesianae, 33 (2019), 276-283. https://doi.org/10.2478/amsil-2018-0009

[9] Szynal-Liana, A., The Horadam hybrid numbers, Discussiones Mathematicae-General Algebra and Applications, 38 (2018), 91-98. https://doi.org/10.7151/dmgaa.1287

[10] Szynal-Liana, A., Włoch, I., On Pell and Pell-Lucas hybrid numbers, Commentationes Mathematicae, 58 (2018), 11-17. https://doi.org/10.14708/cm.v58i1-2.6364

[11] Szynal-Liana, A., Włoch, I., On Generalized Mersenne hybrid numbers, Annales Universitatis Mariae Curie-Sklodowska Lublin-Polonia, (2020), 77-84. https://doi.org/10.17951/ a.2020.74.1.77-84

[12] Szynal-Liana, A., Włoch, I., The Fibonacci hybrid numbers, Util. Math., 110 (2019), 3-10.

[13] Szynal-Liana, A., Włoch, I., Introduction to Fibonacci and Lucas hybrinomials, Complex Variables and Elliptic Equations, 65(10) (2020), 1736-1747. https://doi.org/10.1080/ 17476933.2019 .1681416

[14] Şentürk, TC., Bilgici, G., Daşdemir, A., Ünal, Z., A study on Horadam hybrid numbers, Turkish Journal of Mathematics, 44 (2020), 1212-1221. https://doi.org/10.3906/mat-190877 